\title{
CARACTERIZAÇÃO DE MISTURA ASFÁLTICA ENSACADA PARA UTILIZAÇÃO EM RODOVIAS FEDERAIS
}

\author{
CHARACTERIZATION OF BAGGED ASPHALT MIXTURE FOR USE ON FEDERAL HIGHWAYS
}

\section{Alana Grochowalski Araujoㅁ, Mario Sergio de Souza Almeida ${ }^{2}$, Weiner Gustavo Silva Costa ${ }^{3}$, Jamille Freitas Fiuza ${ }^{4}$, Cleidson Carneiro Guimarães ${ }^{5}$}

\author{
${ }^{1}$ Universidade Federal do Recôncavo da Bahia, Centro de Ciências Exatas e Tecnológicas. alanagrocho@hotmail.com \\ ${ }^{2}$ Universidade Federal do Recôncavo da Bahia, Centro de Ciências Exatas e Tecnológicas. mario.almeida@ufrb.edu.br \\ ${ }^{3}$ Universidade Federal do Recôncavo da Bahia, Centro de Ciências Exatas e Tecnológicas. weiner@ufrb.edu.br \\ ${ }^{4}$ Universidade Federal do Recôncavo da Bahia, Centro de Ciências Exatas e Tecnológicas. fiuzajf@outlook.com \\ ${ }^{5}$ Universidade Federal do Recôncavo da Bahia, Centro de Ciências Exatas e Tecnológicas.cleidsonguimaraes@ufrb.edu.br
}

\section{Recebido:}

28 de janeiro de 2021

Aceito para publicação:

05 de março de 2021

Publicado:

30 de março de 2021

Editor de área:

Georgina L. A. Braga

Palavras-chaves:

Dosagem Marshall.

Concreto Asfáltico.

Pré-Mistura à Frio.

Emulsão Asfáltica

Keywords:

Marshall Dosing.

Asphalt Concrete.

Cold Asphalt Mixture.

Asphalt Emulsion

DOI:

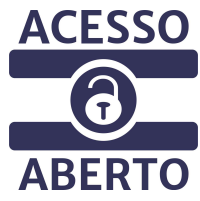

\section{RESUMO}

A elevada demanda por ligantes e misturas asfálticas na construção, manutenção e recuperação de pavimentos rodoviários estimula é inspira pesquisadores de todo o mundo na busca de novas alternativas tecnológicas para produtos asfálticos, de forma a agregar praticidade, economia e qualidade às misturas. Nesse contexto vem ganhando notoriedade no Brasil a utilização de Misturas Asfálticas Ensacadas (MAE) as quais, conforme informação das diferentes marcas existentes no mercado, são misturas fabricadas a quente e com aplicação realizada a frio, tendo como finalidade a sua utilização em reparos de pavimentos (tapa-buraco). Este trabalho teve como objetivo caracterizar uma mistura asfáltica ensacada comercializada no Estado da Bahia e analisá-la comparativamente a duas outras misturas de controle, uma a quente e outra a frio, dosadas em laboratório. Para avaliar a qualidade do produto comercializado determinou-se a granulometria, comportamento mecânico e parâmetros volumétricos da mistura em diferentes idades, através de ensaios da metodologia Marshall visando sua aplicação em rodovias federais. Os resultados preliminares demonstraram que a MAE apresenta comportamento mecânico similar à mistura a frio e distinto da mistura a quente para uso em rodovias federais, mesmo não se enquadrando em faixa granulométrica normatizada. Na avaliação realizada em diferentes idades ficou evidenciada a perda de resistência da mistura comercial, testada como mistura fria ao longo do tempo, provavelmente relacionada à perda precoce das características retardadoras de pega do aditivo utilizado no ligante asfáltico para fabricação do produto, não apresentando comportamento adequado após seis meses de produção. Os resultados demonstraram, ainda, a necessidade de normatização específica e aprimoramento na fabricação do produto.

\section{ABSTRACT}

The high demand for binders and asphalt mixtures in construction, maintenance and recovery of road pavements, stimulates and inspires researchers from all over the world in search of new technological alternatives for asphalt products, in order to add practicality, economy and quality to the mixtures. In this context, the use of Bagged Asphalt Mixtures has been well known in Brazil which, according to information from the different brands on the market, are mixtures manufactured hot and applied cold. Its main purpose is to be used in pavement repairs. This work aimed to characterize a Bagged Asphalt Mixture marketed in the State of Bahia and to compare it with two other control mixtures, one hot and one cold, designed in the laboratory. In order to evaluate the quality of the marketed product, the granulometry, mechanical behavior and volumetric parameters of the mixture at different age groups, were determined through Marshall methodology tests aiming application on federal highways. The preliminary results demonstrate that the MAE presents a mechanical 


\begin{abstract}
behavior similar to the cold mixture and distinct from the hot mixture for use on federal highways, even if it doesn't display the standardized granulometric range. The evaluation carried out at different ages revealed the loss of strength of the commercial mixture, tested as a cold mixture over time, probably related to the early loss of the retarding characteristics of the additive used in the asphalt binder for the manufacture of the product, without showing proper behavior after six months of production. The results also demonstrated a need for specific standardization and improvement in the manufacture of the product.
\end{abstract}

\title{
1. INTRODUÇÃO
}

A predominância do modo rodoviário de transporte de carga no Brasil (CNT, 2019) pereniza no país o uso de misturas asfálticas para a construção e manutenção de pavimentos. Uma tendência atual consiste na pesquisa e desenvolvimento de misturas asfálticas que facilitem a trabalhabilidade, demandem menor consumo energético e que priorizem a minimização de impactos ambientais em seu processo de fabricação (Sena Neto et al., 2019). No ponto central do desenvolvimento de novos produtos relacionados às misturas asfálticas, encontra-se o uso de aditivos os quais promovem características aos ligantes que permitem modificar e aperfeiçoar algumas de suas características (Santos et al., 2015; Santana et al., 2018; Dulaimi et al., 2020; Xavier et al., 2020).

Entretanto, o emprego de misturas a quente em serviços de manutenção e conservação de rodovias, como em operação tapa buraco, apesar do desempenho satisfatório, apresenta algumas dificuldades para sua aplicação em pequena escala, ou mesmo em serviços emergenciais. Sua utilização demanda instalação de usina de maior porte, sofisticado controle tecnológico e mão de obra de maior qualificação para seu manuseio. Dessa forma, para conservação rotineira pontual de pavimentos asfálticos da malha viária federal vem ganhando espaço a ideia de se utilizar misturas que possam ser aplicadas a frio, as quais apresentem maior facilidade de produção, armazenamento, transporte e aplicação, quando comparadas com misturas a quente convencionais. Em busca de um produto prático, eficiente e para uso pontual, algumas empresas vêm ofertando misturas asfálticas, definidas pelos fabricantes como concreto asfáltico usinado a quente estocável, para venda a granel, normalmente embaladas em sacos de $25 \mathrm{~kg}$ para aplicação a frio, se diferenciando, portanto, das misturas a quente convencionais. De acordo com diferentes fabricantes esse material foi desenvolvido a partir da utilização de Cimento Asfáltico de Petróleo (CAP) modificado por aditivo retardador de pega que confere à mistura a condição de ao esfriar não endurecer e assim permanecer por até 24 meses se devidamente acondicionado. O inconveniente desse produto atualmente é a falta de normatização que forneça parâmetros para controle na aplicação em rodovias de médio e alto volume de tráfego, característicos de rodovias federais.

Santana et al. (2017) avaliaram a qualidade de amostras de Misturas Asfálticas Ensacadas (MAE) de três fabricantes de diferentes estados do País. A amostra do primeiro fabricante foi usinada a quente com CAP 50/70 modificado com um retardador de cura para propiciar a estocagem e aplicação a frio. A amostra do segundo fabricante tratou-se de um concreto asfáltico constituído por pedra britada e CAP, aprimorado com resíduos sólidos de pneus para propiciar maior resistência, impermeabilidade e aderência à mistura. Do terceiro fabricante foram analisadas duas amostras de lotes diferentes. As amostras foram testadas como concreto asfáltico, a partir de ensaios laboratoriais de estabilidade e fluência Marshall, Resistência à Tração por compressão diametral (RT), além da determinação do Volume de vazios (Vv) e Relação Betume/Vazios (RBV). Os ensaios de Módulo de Resiliência (MR) inicialmente previstos não puderam ser realizados na maioria das amostras porque os corpos de provas romperam assim que foram posicionados na prensa antes mesmo da aplicação de carga. O ensaio com corpos de prova da Amostra 3A moldados pela Metodologia Superpave foi o único que pôde ser realizado. Os resultados obtidos das amostras compactadas revelaram 
grande heterogeneidade entre os diferentes produtos, com elevados Coefícientes de Variação (CV) em relação aos respectivos valores médios, com destaque para: i) Vv (média $=9,54 \%$ e CV =73,80\%); ii) RBV (média $=66,73 \%$ e $\mathrm{CV}=35,24 \%$ ); iii) estabilidade (média $=118,18 \mathrm{kgf}$ e $\mathrm{CV}=50,02 \%$ ); iv) fluência (média $=1,71 \mathrm{~mm}$ e CV $=12,65 \%$ ) e; v) RT (média $=0,031 \mathrm{kgf} / \mathrm{cm} 2$ e CV $=104,59 \%$ ). O resultado do MR médio da Amostra 3B foi de $660 \mathrm{kgf} / \mathrm{cm} 2$, considerado muito baixo para misturas asfálticas. Os autores constataram que os produtos não alcançaram os requisitos mínimos exigidos pela especificação de serviço do DNIT 31 (DNIT, 2006a), tampouco a qualidade alegada pelos fabricantes.

Rezende et al. (2019) caracterizaram o produto denominado pelos autores por Concreto Asfáltico Estocável (CAE). Essa mistura asfáltica, ainda segundo os autores, vem sendo utilizada sem especificação técnica no Estado de Goiás, Brasil, oportunizando uma demanda pela análise de seu comportamento físico e mecânico. O produto foi recebido no laboratório em recipientes plásticos de $200 \mathrm{~kg}$, oriunda de fabricante anônimo. Para viabilizar a compactação dos corpos de prova utilizados nos ensaios mecânicos pela metodologia Superpave, os autores seguiram o mesmo procedimento adotado por Santana et al. (2017) onde a amostra estudada foi previamente aquecida a $600 \mathrm{C}$. Foram moldados 12 (doze) corpos de prova separados em duas condições: seis com cura e seis sem cura. Cada configuração produzida foi ensaiada para determinação da RT e MR. Os resultados médios de RT de $0,48 \mathrm{kgf} / \mathrm{cm} 2$, com cura, e $0,50 \mathrm{kgf} / \mathrm{cm} 2$, sem cura, demonstraram pouca influência desse tratamento testado. Entretanto, comparativamente aos resultados de Santana et al. (2017), os valores de RT apesar de, na média, serem superiores e com CV bem menor, da ordem de 5,5\%, ainda assim estão muito abaixo da exigência mínima da especificação DNIT 31 (DNIT, 2006a) que é de $6,5 \mathrm{kgf} / \mathrm{cm} 2$. Os resultados de MR também foram pouco influenciados pelo tempo de cura das amostras com valores obtidos nos dois tratamentos da ordem de $23.000 \mathrm{kgf} / \mathrm{cm} 2$. Dessa forma, os resultados obtidos por Rezende et al. (2019) evidenciaram comportamento mecânico inferior da mistura comercial comparado ao de concretos asfálticos convencionais utilizados no Estado de Goiás, mostrando assim que o material não se comporta como prometem os fabricantes.

Neste contexto, o presente trabalho buscou estudar o comportamento de uma mistura asfáltica ensacada comercial com vistas na aplicação em obras de manutenção de pavimentos de rodovias federais, de acordo com as normas de misturas asfálticas vigentes no Departamento Nacional de Infraestrutura de Transportes (DNIT).

\section{METODOLOGIA}

O programa experimental teve início com o recebimento da mistura ensacada, doada por um fabricante local, a qual passou pelo processo de caracterização compreendendo a determinação do teor de ligante e granulometria da mistura. Posteriormente, o comportamento mecânico do produto foi avaliado através de ensaios de estabilidade e fluência Marshall, conjuntamente com a verificação da volumetria da mistura compactada.

Em seguida, foram caracterizados o Cimento Asfáltico de Petróleo (CAP), emulsão asfáltica e agregados para dosagem de misturas asfálticas de controle, a quente e a frio, as quais serviram de base de comparação na classificação da mistura ensacada de acordo com as especificações de serviço do DNIT 31 (DNIT, 2006a) e 153 (DNIT, 2010a). Por fim, o produto comercial foi ensaiado em diferentes idades de fabricação como uma mistura a frio (DNIT, 2010a) para avaliação do comportamento ao longo do tempo de armazenamento. 


\subsection{Materiais}

A Mistura Asfáltica Ensacada (MAE) utilizada no presente estudo é um dos produtos comercializados no estado da Bahia. Foram fornecidas oito amostras do produto, acondicionadas em sacos de $25 \mathrm{~kg}$ cada, as quais foram separadas em quatro lotes distintos, ou seja, dois sacos por lote, todos com idades entre um e dois meses de fabricação. Três lotes foram utilizados para caracterização da mistura e o quarto foi destinado a execução de ensaios em diferentes idades de fabricação.

Também foram obtidos com o fabricante $100 \mathrm{~kg}$ de cada agregado utilizado na fabricação do produto (brita 3/8" e areia artificial de britagem, conhecida como pó de pedra), originados da pedreira Paisartt, localizada no município de Lauro de Freitas/BA, os quais foram usados no traço do Concreto Asfáltico de controle (CACONT). Complementarmente, foram adquiridos no mercado local de Cruz das Almas/BA 100 kg de brita 5/8" e $100 \mathrm{~kg}$ de pó de pedra, oriundos da Pedreira Pedra do Cavalo, município de Muritiba/BA, para uso na elaboração de um traço de Pré-Misturado a Frio de controle (PMFCONT).

\subsection{Caracterização da Mistura Asfáltica Ensacada}

\subsubsection{Determinação do Teor de Ligante e Granulometria}

A extração do ligante asfáltico da mistura ensacada foi realizada com o objetivo de determinar a quantidade de CAP residual e a composição granulométrica da mistura. Para a extração foram utilizados o Rotarex, método DNER 53 (DNER, 1994a), e Soxhlet, método DNIT 158 (DNIT, 2011). Para o Rotarex utilizou-se três amostras representativas da mistura ensacada para cada lote destinado aos ensaios de caracterização, com aproximadamente $500 \mathrm{~g}$ cada, ensaiadas individualmente para a determinação da percentagem média de ligante asfáltico em cada lote. Para confirmação do percentual de ligante encontrado no Rotarex, ensaiouse uma amostra representativa para cada lote, também de $500 \mathrm{~g}$, no Soxhlet. O solvente utilizado para a realização de ambos os ensaios foi o tricloroetileno.

Em seguida, os agregados obtidos em cada processo de extração anteriormente descrito, foram colocados para secagem em estufa, à temperatura entre $105^{\circ} \mathrm{C}$ e $110^{\circ} \mathrm{C}$, até constância de massa. De posse dos agregados secos foram realizados ensaios de granulometria de acordo com o método do DNER 83 (DNER, 1998a). A granulometria da MAE está apresentada na Tabela 1.

\subsubsection{Caracterização Marshall da MAE}

A caracterização mecânica da mistura ensacada foi realizada com base na metodologia Marshall para CA (DNER, 1995) e para PMF, (DNER, 1994b). Os corpos de prova (CPs) foram compactados com 75 golpes em cada face, sendo três CPs para cada lote. As amostras ensaiadas como concreto asfáltico foram rompidas na prensa Marshall 24h após a compactação, enquanto as ensaiadas como PMF foram rompidas 48h após a compactação. Em função do quantitativo reduzido do produto os ensaios de resistência à tração (DNIT, 2018) não foram realizados nessa fase de caracterização do produto.

Vale destacar as difículdades de aplicação do método 43 do DNER (DNER, 1995), na mistura ensacada, principalmente com relação ao aquecimento dos CPs, antes da ruptura, com uso do banho maria. Ao serem aquecidos entre 30 e 40 min com esse equipamento a 60 0C, os corpos de prova apresentaram pouca ou nenhuma resistência à submersão em água, chegando a desagregarem-se. A solução encontrada foi substituir o método de aquecimento pela estufa, também previsto na referida norma, com amostras mantidas na mesma temperatura do banho maria pelo tempo de $2 \mathrm{~h}$. 
Importante destacar, ainda, que diferentemente de Santana et al. (2017) e Rezende et al. (2019) não foi realizado aquecimento prévio da mistura ensacada a 60 0C para compactação das amostras destinadas aos ensaios Marshall. Esse procedimento altera as condições reais de compactação da mistura no campo. A dificuldade de desmoldagem dos CPs relatada pelos autores foi contornada com lubrificação dos moldes metálicos com vaselina em pasta antes da compactação.

Tabela 1: Granulometria dos agregados da MAE após extração do ligante

\begin{tabular}{cccccccccc}
\hline \multicolumn{3}{c}{ Peneiras } & \multicolumn{4}{c}{ Rotarex (\%) } & \multicolumn{4}{c}{ Soxhlet (\%) } \\
\hline Nomenclatura & $\begin{array}{c}\text { Abertura } \\
(\mathrm{mm})\end{array}$ & Lote 1 & Lote 2 & Lote 3 & Média & Lote 1 & Lote 2 & Lote 3 & Média \\
\hline $1 / 2 "$ & 12,7 & 100 & 100 & 100 & 100 & 100 & 100 & 100 & 100 \\
\hline $3 / 8 "$ & 9,5 & 99,76 & 98,67 & 99,53 & 99,32 & 99,62 & 95,11 & 100 & 98,24 \\
\hline$\# 4$ & 4,8 & 84,20 & 56,43 & 77,24 & 72,62 & 81,95 & 77,16 & 86,11 & 81,74 \\
\hline$\# 10$ & 2,0 & 53,98 & 38,23 & 54,86 & 49,02 & 50,85 & 47,37 & 64,89 & 54,37 \\
\hline$\# 40$ & 0,42 & 24,15 & 20,02 & 28,17 & 24,11 & 23,53 & 25,71 & 31,23 & 26,82 \\
\hline$\# 80$ & 0,175 & 14,45 & 10,34 & 14,81 & 13,20 & 15,39 & 13,36 & 17,20 & 15,32 \\
\hline$\# 200$ & 0,075 & 8,56 & 5,73 & 8,35 & 7,55 & 8,04 & 6,88 & 8,96 & 7,96 \\
\hline
\end{tabular}

\subsection{Dosagem dos Traços de controle CACONT e PMFCONT}

\subsubsection{Caracterização dos Agregados para os Traços}

A próxima etapa experimental consistiu em projetar dois traços de misturas asfálticas de controle, uma a quente (CACONT) e outra a frio (PMFCONT), para avaliação da MAE. Para isso, determinou-se a granulometria dos agregados disponibilizados pela empresa (brita 3/8"e pó de pedra), sendo estes os mesmos utilizados na fabricação da mistura asfáltica ensacada.

Para a dosagem de um traço de PMFCONT considerou-se características de misturas a frio rotineiramente utilizadas em obras de manutenção e conservação de pavimentos no Estado da Bahia. Para isso, foi dosado um traço do PMF de acordo com a especificação DNIT 153 (DNIT, 2010a), considerando o uso de agregados locais por serem materiais regularmente utilizados na região para confecção de misturas asfálticas convencionais (fria e quente). Utilizou-se para isso dois agregados, a brita 5/8", como agregado graúdo, e o pó de pedra, como agregado miúdo, ambos oriundos da Pedreira Pedra do Cavalo, localizada na cidade de Muritiba, estado da Bahia, originados de rocha Granodiorito de médio porte (DNPM, 2018). Com esses dois agregados foi possível enquadrar a mistura na faixa granulométrica $\mathrm{D}$, a mais densa e utilizada na prática dentre as quatro faixas prescritas na especificação DNIT 153 (DNIT, 2010a). A escolha dessa faixa foi em função das principais aplicações de PMFs concentrarem-se em serviços de revestimento e reparos localizados, onde misturas mais "finas" (ou densas) oferecem melhor trabalhabilidade e acabamento, sem perda de qualidade estrutural.

Os ensaios de granulometria (Tabela 2) foram realizados conforme o método DNER 83 (DNER, 1998a) nos agregados graúdos (britas 3/8" e 5/8") e miúdos (pó de pedra fornecido pelo fabricante da MAE e adquirido no mercado local), sendo três amostras para cada material com, aproximadamente, 1,5 $\mathrm{kg}$ para cada amostra. Vale destacar que as peneiras utilizadas nesse ensaio para cada agregado foram as exigidas pela especificação DNIT 31 (DNIT, 2006a), para concreto asfáltico, e DNIT 153 (DNIT, 2010a), para prémisturado a frio. 
Revista

PAVIMENTAÇÃO

www.revistapavimentacao.org.br

Tabela 2: Granulometria dos agregados das misturas de controle

\begin{tabular}{cccccc}
\hline \multirow{2}{*}{ Peneiras } & & \multicolumn{2}{c}{ Agregados para o CACONT } & \multicolumn{2}{c}{ Agregados para o PMF CONT } \\
\cline { 2 - 6 } Nomenclatura & $\begin{array}{c}\text { Abertura } \\
(\mathrm{mm})\end{array}$ & $(\%)$ & $(\%)$ & $(\%)$ & Pó de pedra \\
\hline $3 / 4 "$ & 19,0 & 100 & 100 & 100 & 100 \\
\hline $1 / 2 "$ & 12,7 & 100 & 100 & 91,72 & 100 \\
\hline $3 / 8 "$ & 9,5 & 93,54 & 98,52 & 40,26 & 99,79 \\
\hline$\# 4$ & 4,8 & 31,83 & 96,73 & 2,93 & 95,77 \\
\hline$\# 10$ & 2,0 & 4,81 & 76,04 & 1,68 & 59,27 \\
\hline$\# 40$ & 0,42 & 3,05 & 39,78 & - & - \\
\hline$\# 80$ & 0,175 & 2,56 & 25,45 & - & - \\
\hline$\# 200$ & 0,075 & 1,87 & 15,10 & 0,54 & 12,78 \\
\hline
\end{tabular}

O ensaio de Equivalente de Areia (EA) para os agregados miúdos foi realizado de acordo com o método DNER 54 (DNER, 1998b). Para sua realização foram obtidas três amostras representativas de material passando na peneira de $4,8 \mathrm{~mm}$, com cerca de $110 \mathrm{~g}$ cada amostra. A determinação da densidade real do agregado graúdo foi segundo o método DNER 81 (DNER, 1998c) e para a densidade real do agregado miúdo foi de acordo com o método DNER 84 (DNER, 1998d). Os ensaios foram realizados em triplicata para definição de um valor médio. O ensaio de adesividade do agregado ao ligante asfáltico foi realizado com o agregado graúdo conforme o método DNER 78 (DNER, 1994c). Os resultados estão apresentados na Tabela 3.

Tabela 3: Caracterização dos agregados das misturas de controle

\begin{tabular}{ccccc}
\hline \multirow{2}{*}{ Ensaio } & \multicolumn{2}{c}{ Agregados para o CACONT } & \multicolumn{2}{c}{ Agregados para o PMF CONT } \\
\cline { 2 - 5 } & Brita 3/8” & Pó de pedra & Brita 5/8” & Pó de pedra \\
\hline Densidade aparente & 2,750 & - & 2,705 & - \\
\hline Densidade real & 2,760 & 2,750 & 2,724 & 2,857 \\
\hline Absorção (\%) & 0,45 & - & 0,25 & - \\
\hline Adesividade & Satisfatória & - & Satisfatória & - \\
\hline $\begin{array}{c}\text { Equivalente de areia } \\
(\%)\end{array}$ & - & 80,07 & & 63,72 \\
\hline
\end{tabular}

\subsubsection{Caracterização do Cimento Asfáltico de Petróleo e Emulsão Asfáltica}

O ligante asfáltico utilizado na dosagem do traço do CACONT foi o CAP 50/70 convencional e para sua caracterização foram realizados os ensaios de Penetração, método DNIT 155 (DNIT, 2010b), Viscosidade Saybolt-Furol, método DNER 4 (DNER, 1994d), Ponto de Fulgor, método 148 (DNER, 1994e), e Ponto de Amolecimento Anel e Bola, método DNIT 131 (DNIT, 2010c). Nesse estudo, considerou-se a densidade do CAP fornecida pelo fornecedor do ligante asfáltico (Brasquímica Produtos Asfálticos Ltda.) igual à 1,03 $\mathrm{g} / \mathrm{cm}^{3}$.

Destaca-se que em função das características do ligante asfáltico com aditivo utilizado na produção da mistura ensacada, conforme relato dos fabricantes, utilizou-se um lote de CAP 50/70 para confecção do CACONT que apresentou a menor consistência possível, próximo aos limites estabelecidos pelas normas, 
conforme os valores apresentados na Tabela 4, com destaque para a baixa viscosidade observada a $1770 \mathrm{C}$ e o baixo ponto de amolecimento (0C), ambos inferiores aos limites mínimos estabelecidos pela especificação de material DNIT 95 (DNIT, 2006b), os quais ratificam a baixa consistência do CAP utilizado.

Em função da escolha da faixa D para o traço do PMFCONT, a emulsão asfáltica utilizada foi do tipo RL-1C, que é de ruptura lenta, relacionada a granulometria mais densa do traço, conforme a especificação DNIT 153 (DNIT, 2010a). Os ensaios de caracterização da emulsão foram realizados de acordo com a especificação de material DNIT 165 (DNIT, 2013), cujos limites estabelecidos devem ser atendidos pelo produto. Na Tabela 5 são apresentados os resultados nos ensaios de caracterização da emulsão asfáltica, realizados pela própria empresa fabricante e fornecedora do produto (Brasquímica Produtos Asfálticos Ltda.).

Tabela 4: Caracterização do CAP 50/70

\begin{tabular}{|c|c|c|c|c|}
\hline Parâmetro & & $\begin{array}{c}\text { Limites } \\
\text { DNIT (2006b) }\end{array}$ & Resultados & Método de ensaio \\
\hline Penetração $(0,1 \mathrm{~mm})$ & & $50-70$ & 70 & DNIT-ME 155/2010 \\
\hline \multirow{3}{*}{ Viscosidade (s) } & $135^{0} \mathrm{C}$ & Min. 141 & 159 & \multirow{3}{*}{ DNER-ME 4/1994 } \\
\hline & $150{ }^{\circ} \mathrm{C}$ & Min. 50 & 69 & \\
\hline & $177{ }^{0} \mathrm{C}$ & $30-150$ & 28,5 & \\
\hline Ponto de fulgor $\left({ }^{0} \mathrm{C}\right)$ & & Min. 235 & 335 & DNER-ME 148/1994 \\
\hline $\begin{array}{l}\text { Ponto de amolecimento } \\
\qquad\left({ }^{0} \mathrm{C}\right)\end{array}$ & & 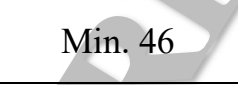 & 39,5 & DNIT-ME 131/2010 \\
\hline
\end{tabular}

Tabela 5: Caracterização da emulsão asfáltica RL-1C

\begin{tabular}{cccc}
\hline Parâmetro & $\begin{array}{c}\text { Limites } \\
\text { DNIT (2013) }\end{array}$ & Resultados & Método de ensaio \\
\hline Viscosidade Saybolt & Máx 90 & 18 & NBR 14491 \\
\hline Sedimentação após 5 & Máx 5 $(\% \mathrm{~m} / \mathrm{m})$ & 2,4 & NBR 6570 \\
\hline Peneiração $(0,84 \mathrm{~mm})$ & Máx 0,1 $(\% \mathrm{~m} / \mathrm{m})$ & 0,01 & NBR 14393 \\
\hline Carga de partícula $(+/-$ & Positiva & Positiva & NBR 6567 \\
\hline pH & Máx 6,5 & 2,6 & NBR 6299 \\
\hline Resíduo de asfalto & Mín 60 $(\% \mathrm{~m} / \mathrm{m})$ & 60,2 & NBR 14376 \\
\hline
\end{tabular}

\subsubsection{Determinação do teor de ligante asfáltico de projeto dos traços de controle}

Como um dos objetivos do presente estudo foi determinar se a mistura ensacada se comporta, efetivamente, como uma mistura a quente, conforme é divulgado pelos fabricantes, a partir do conhecimento da granulometria dos agregados obtidos do processo de extração (Tabela 1), projetou-se uma composição granulometricamente similar à utilizada na fabricação comercial, composta dos mesmos agregados recebidos do fabricante, de forma que a mistura produzida em laboratório fosse a mais fiel possível à original do produto, diferenciando-se apenas pelo tipo de ligante asfáltico utilizado.

Dessa forma, de posse da granulometria dos agregados recebidos (Tabela 2), definiu-se a combinação entre eles que melhor se ajustou a granulometria do ensacado, combinação essa constituída por $33 \%$ de brita $3 / 8$ " e $67 \%$ de pó de pedra. Importante salientar que à granulometria da mistura ensacada que 
serviu de base para a elaboração do traço de CACONT foi a determinada após os ensaios de extração com o Soxhlet.

O comparativo entre o traço do CACONT e a granulometria da MAE após extração está apresentado na Figura 1(a). Da mesma forma, foi possível definir o percentual de cada agregado adquirido no mercado local na mistura, para atendimento à faixa D da especificação DNIT 153 (DNIT, 2010a) e confecção do traço do pré-misturado a frio de controle (PMFCONT). A melhor combinação entre os dois agregados correspondeu a $65 \%$ de brita $5 / 8$ " e $35 \%$ de pó de pedra. A composição granulométrica do traço está detalhada na Figura 1(b), incluindo a tolerância por peneira estabelecida pela mesma norma.

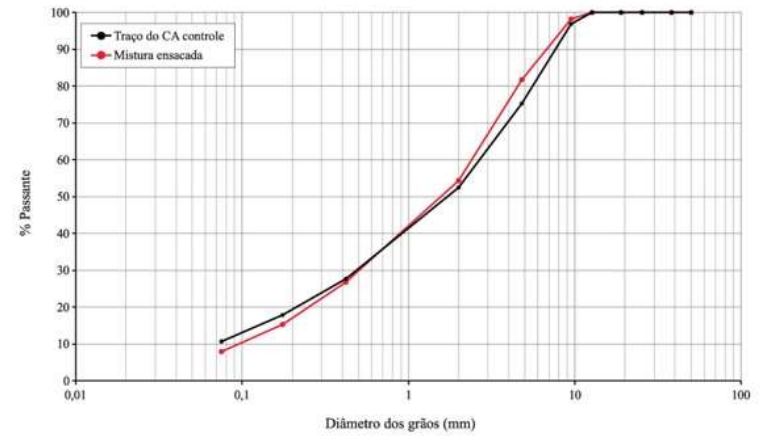

(a) Granulometria da MAE e do traço do CACONT

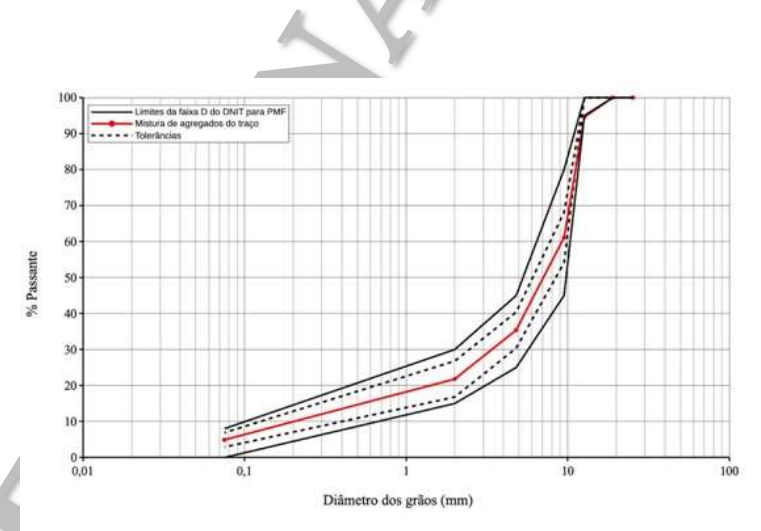

(b) Granulometria do traço do $\mathrm{PMF}_{\mathrm{CONT}}$

Figura 1: Granulometrias das misturas MAE, CAcont e PMFCONT

Como no presente estudo busca-se caracterizar a mistura ensacada para utilização em rodovias federais, a determinação do teor ótimo de CAP do traço do CACONT foi realizada considerando tráfego pesado, ou seja, com aplicação de energia de compactação de 75 golpes. Dessa forma, foram testados os teores de 4,0; 4,5; 5,0; 5,5 e 6,0\% de CAP 50/70, com três corpos de prova (CPs) para cada teor, seguindo a metodologia Marshall definida pelo método de ensaio DNER 43 (DNER, 1995). De forma a obter um PMFCONT para comparação com a mistura ensacada, o estudo do traço também foi elaborado para apenas uma energia de compactação do método DNER 107 (DNER, 1994b), com 75 golpes. Para a determinação do teor de projeto de ligante asfáltico do traço do PMFCONT foram testados os teores de 4,0; 4,5; 5,0; 5,5 e $6,0 \%$ de CAP residual, que correspondem, respectivamente, a $6,7 \%, 7,5 \%, 8,3 \%, 9,2 \%$ e $10 \%$ de emulsão asfáltica, considerando o resíduo de asfalto determinado na Tabela 4. Logo após foram confeccionados para ambas as misturas três corpos de prova (CPs) para cada teor, seguindo a metodologia Marshall definida pelos métodos DNER 43 e 107 (DNER, 1995; DNER, 1994b). A massa de mistura preparada para cada CP foi de, aproximadamente, $1200 \mathrm{~g}$.

Em seguida, para ambas as misturas, foram medidas as dimensões dos corpos de prova para a determinação dos parâmetros volumétricos do traço, com aplicação do método DNER 117 (DNER, 1994f). Posteriormente, os CPs foram ensaiados de acordo com o método DNER 43 (DNER, 1995), para o CA, e DNER 107 (DNER, 1994b), para PMF, na prensa para determinação da estabilidade Marshall, fluência, e calculados Volume de vazios (Vv) e Relação Betume/Vazios (RBV) de cada mistura, cujos valores médios estão apresentados na Figura 2.

Dentre os teores testados o de projeto deverá ser aquele que obtiver a maior massa específica aparente, ou seja, o menor volume de vazios, atendendo aos demais limites estabelecidos pelas 


\section{Revista PAVIMENTAÇÃO}

especificações DNIT 31 (DNIT, 2006), para CA, e 153 (DNIT, 2010a), para PMF. Dessa forma, para o CA, considerando-se os resultados de estabilidade, fluência, Vv e RBV apresentados na Figura 2 (a, b, c e d), dentre os teores de CAP testados, apenas o de 5,5\% atendeu aos limites exigidos pela especificação DNIT 31 (DNIT, 2006) para os quatro atributos. Assim, o traço de projeto do CACONT, considerando o ligante asfáltico, ficou definido com 5,5\% de CAP, $31,19 \%$ de brita $3 / 8$ " e $63,31 \%$ de pó de pedra.

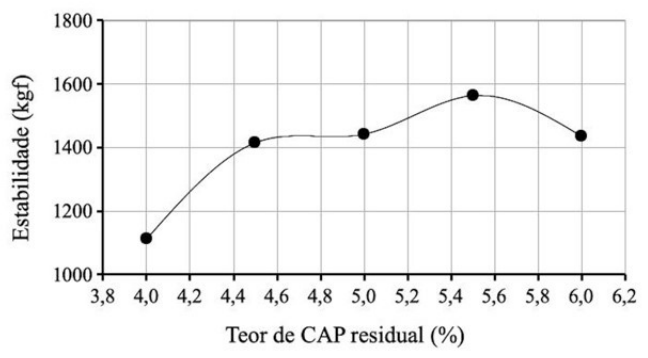

(a) $\mathrm{CA}_{\mathrm{CONT}}$ - Estabilidade

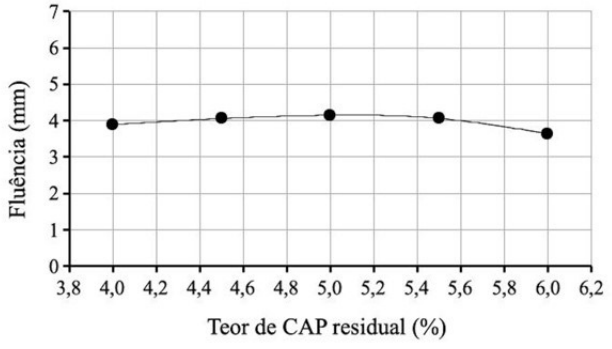

(b) CA CONT - Fluência

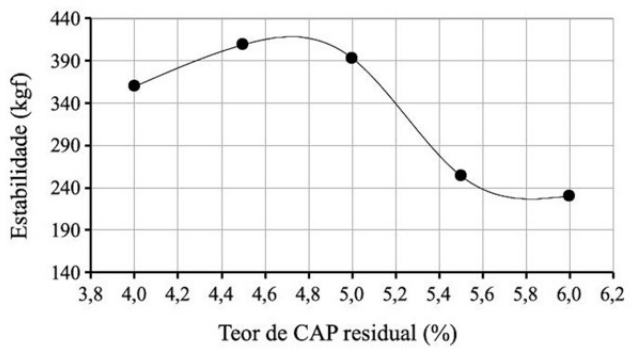

(e) $\mathrm{PMF}_{\text {CONT }}$ - Estabilidade

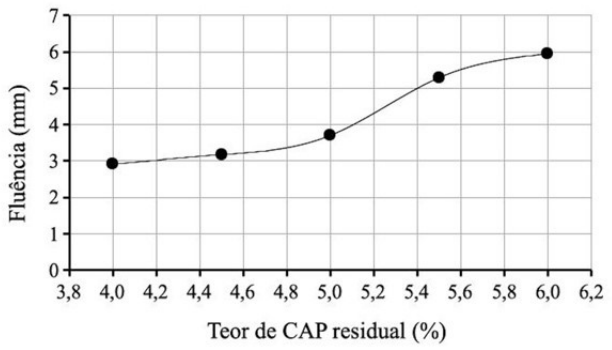

(f) $\mathrm{PMF}_{\mathrm{CONT}}$ - Fluência

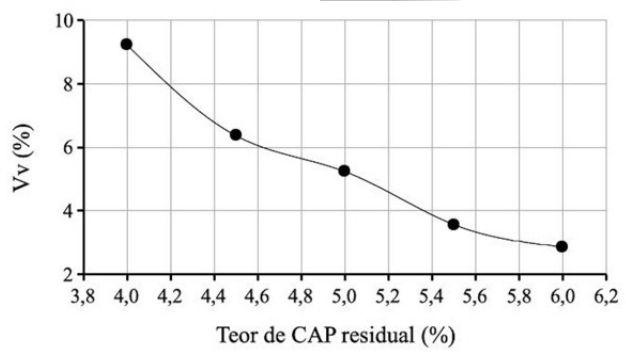

(c) $\mathrm{CA}_{\text {CONT }}$ - Volume de vazios

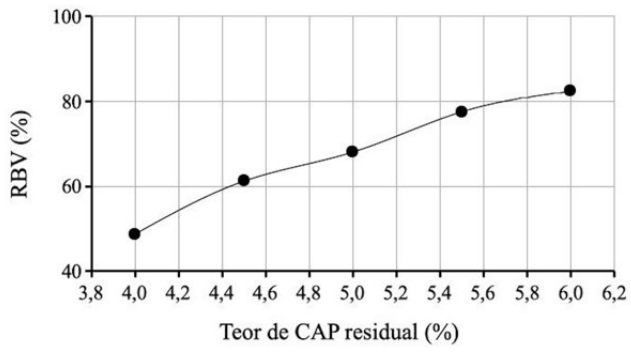

(d) $\mathrm{CA}_{\mathrm{CONT}}$ - Relação betume/vazios

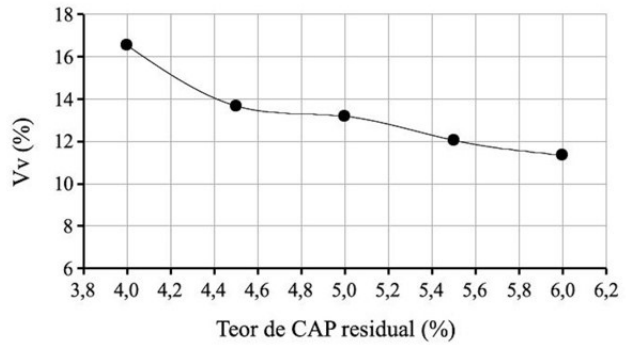

(g) $\mathrm{PMF}_{\mathrm{CONT}}$ - Volume de vazios

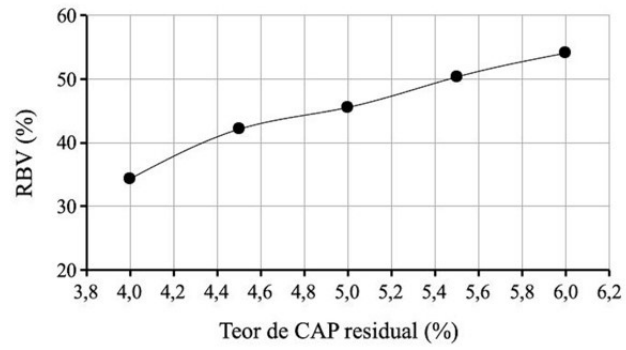

(h) $\mathrm{PMF}_{\mathrm{CONT}}$ - Relação betume/vazios

Figura 2: Determinação dos teores de projeto dos traços das misturas controle 
Para o PMF, também se considerando os resultados de estabilidade, fluência e Vv apresentados na Figura 2 (e, f e g), dentre os teores testados, os percentuais de 4\%, 4,5\% e 5\% de CAP residual atenderam aos limites exigidos pela especificação DNIT 153 (DNIT, 2010a) para os três atributos. Dentre estes o teor de $5 \%$ foi o que obteve a maior massa especifica aparente. Entretanto, entre $4,5 \%$ e $5 \%$ houve queda na estabilidade, motivo pelo qual optou-se pela média entre estes dois valores. Com essa metodologia adotada, determinou-se o teor de 4,8\% como o de projeto, que equivale a $8 \%$ de emulsão asfáltica, sendo o resíduo de asfalto na emulsão de $60 \%$, conforme a Tabela 4. O traço de projeto do PMFCONT, considerando o ligante asfáltico, ficou definido com 4,8\% de CAP residual ( $8 \%$ de emulsão), 61,88\% de brita 5/8" e 33,32\% de pó de pedra.

\subsection{Controle de qualidade da mistura ensacada}

Para avaliar o comportamento mecânico da mistura comercial em função do tempo de fabricação e acondicionamento em sacos, o produto foi testado como uma mistura a frio e submetido aos ensaios definidos na metodologia Marshall, método DNER 107 (DNER, 1994b), em diferentes idades, além de ensaios de tração por compressão diametral, método DNIT 136 (DNIT, 2018). As amostras recebidas foram acondicionadas em um contêiner plástico dentro de seus respectivos sacos, de modo a minimizar as perdas de umidade (aditivo) no armazenamento ao longo do tempo. A cada idade foram amostrados cerca de 10 kg para determinação, em triplicata, da estabilidade, fluência e volume de vazios de corpos de prova compactados com 75 golpes, ensaiados com idades de fabricação de 30, 90, 180 e 360 dias. Com os resultados obtidos da análise temporal da mistura ensacada foi possível avaliar a variabilidade estatística de cada parâmetro, através do desvio padrão (DP) e coeficiente de variação $(\mathrm{CV})$, os quais serviram como indicativo da qualidade da mistura em função do tempo de acondicionamento do material para venda a granel.

\section{RESULTADOS E DISCUSSÕES}

\subsection{Teor de ligante e granulometria da mistura asfáltica ensacada}

Os teores de ligante da mistura ensacada, obtidos com as técnicas do Rotarex e Soxhlet, estão apresentados na Tabela 6.

Tabela 6: Teor de CAP da mistura ensacada

\begin{tabular}{cccc}
\hline Método & Lote 1 & Lote 2 & Lote 3 \\
\hline Rotarex & 4,55 & 4,55 & 6,08 \\
\hline Soxhlet & 4,52 & 4,57 & 6,13 \\
\hline
\end{tabular}

Observa-se da Tabela 6 que os teores medidos com o Rotarex e Soxhlet apresentaram valores bem semelhantes para cada lote avaliado. Os teores medidos nos três lotes se enquadram nos limites estabelecido pela especificação DNIT 31 (DNIT, 2006a) para concreto asfáltico (4,5\% a 9\%). Por outro lado, ao analisarse com base na especificação DNIT 153 (DNIT, 2010a), para PMF, que estabelece os teores de CAP residual entre $4 \%$ e $6 \%$, os lotes 1 e 2 se enquadram na referida especificação, enquanto o lote 3 ficou ligeiramente superior.

Destaca-se, ainda, a variação observada de teor de ligante do lote 3 em relação aos outros dois lotes, conforme também observado por Santana et. al. (2017). Muito provavelmente esse aumento tenha relação a falhas de controle no processo de fabricação. 


\section{Revista PAVIMENTAÇÃO}

A granulometria da MAE, obtida após extração do ligante asfáltico através do Soxhlet, está apresentada na Figura 3, junto com a faixa de concreto asfáltico do DNIT (2006a) e de PMF do DNIT (2010a).

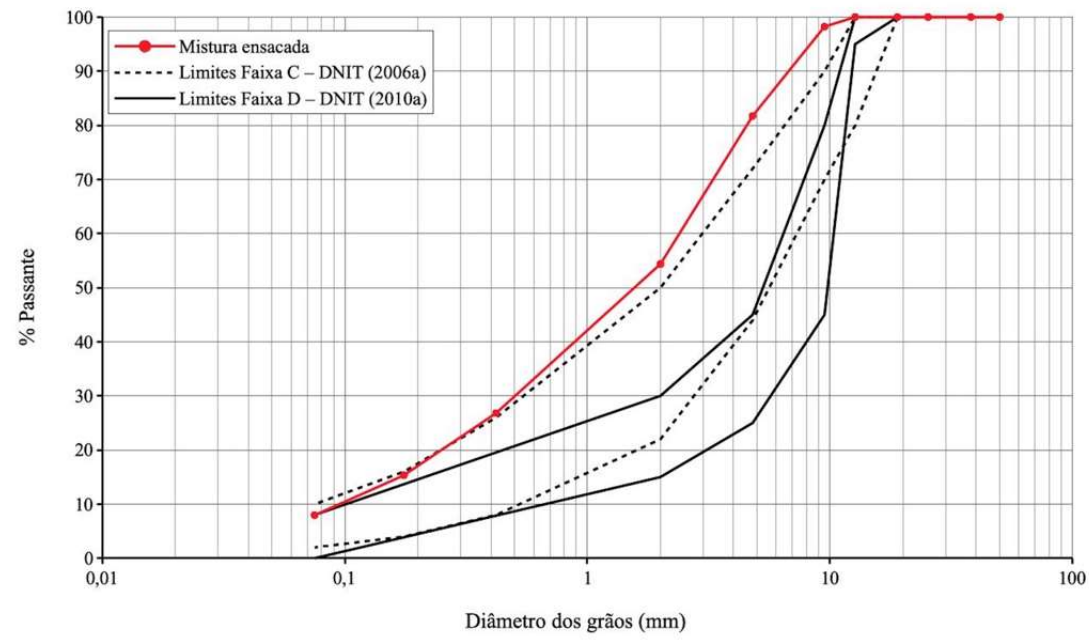

Figura 3: Enquadramento granulométrico da mistura ensacada

É possível observar da Figura 3 que a granulometria encontrada da mistura ensacada ficou fora das faixas do DNIT para misturas tanto a quente quanto a frio. Portanto, do ponto de vista granulométrico, a mistura comercial testada não atende às exigências de concreto asfáltico, conforme a especificação DNIT 31 (DNIT, 2006a), nem tão pouco de PMF, de acordo com a especificação DNIT 153 (DNIT, 2010a), para utilização em rodovias federais.

\subsection{Caracterização Marshall da MAE}

Na Tabela 7 são apresentados os resultados médios de estabilidade Marshall, fluência, Vv e RBV de cada lote analisado, segundo DNER 43 (DNER, 1995) e DNER 107 (DNER, 1994b), para caracterização da mistura ensacada como mistura a quente (CA) e a frio (PMF), respectivamente. São apresentados também os valores de média e Coeficiente de Variação (CV) de cada parâmetro.

Tabela 7: Resultados da caracterização Marshall da mistura ensacada

\begin{tabular}{ccccccc}
\hline Tipo de teste & Parâmetro & Lote 1 & Lote 2 & Lote 3 & Média & CV (\%) \\
\hline \multirow{3}{*}{$\begin{array}{c}\text { Mistura a quente } \\
\text { DNIT 43 }\end{array}$} & Estabilidade (kgf) & 194,56 & 128,30 & 162,01 & 161,62 & 20,50 \\
\cline { 2 - 6 } & Fluência (mm) & 3,05 & 3,89 & 3,82 & 3,59 & 12,99 \\
\cline { 2 - 6 } & Vv (\%) & 12,70 & 6,16 & 6,16 & 8,73 & 39,99 \\
\cline { 2 - 6 } & RBV (\%) & 43,80 & 69,43 & 69,43 & 57,41 & 22,45 \\
\hline \multirow{3}{*}{$\begin{array}{c}\text { Mistura a frio } \\
\text { DNIT 107 }\end{array}$} & Estabilidade (kgf) & 457,49 & 295,69 & 302,44 & 351,87 & 26,01 \\
\cline { 2 - 6 } & Fluência (mm) & 4,30 & 4,30 & 4,06 & 4,22 & 3,28 \\
\cline { 2 - 6 } & Vv (\%) & 12,86 & 7,96 & 6,97 & 9,26 & 34,05 \\
\cline { 2 - 6 } & RBV (\%) & 43,40 & 56,84 & 66,60 & 55,61 & 20,95 \\
\hline
\end{tabular}


A partir dos resultados da Tabela 7 foi possível observar que a mistura ensacada apresentou considerável variação nos resultados, com CVs elevados, em todos os parâmetros analisados quando o produto foi testado como mistura a quente. Resultados similares foram observados por Santana et al. (2017). Essa evidência se repetiu quando os testes consideraram a mistura como do tipo a frio, com exceção, nesse caso, para a fluência que apresentou $\mathrm{CV}<5 \%$. Nota-se que a estabilidade média alcançada com a mistura testada a frio foi $118 \%$ superior ao teste a quente. Entretanto, o comparativo entre os valores medidos de fluência não apresentou diferença que possa ser considerada significativa. No caso dos parâmetros volumétricos, ou seja, volume de vazios e relação betume/vazios, os quais dependem do traço e compactação, também não apresentaram diferenças consideráveis.

\subsection{Avaliação comparativa entre as misturas}

A Tabela 8 apresenta de forma comparativa os resultados médios de estabilidade, fluência, Vv e RBV da MAE, ensaiada a quente e a frio, do CACONT e do PMFCONT, junto com as respectivas exigências das especificações DNIT 31 (DNIT, 2006a) e DNIT 153 (DNIT, 2010a).

Tabela 8: Resultados da caracterização Marshall da mistura ensacada

\begin{tabular}{ccccccc}
\hline Parâmetro & MAE a quente & CA $_{\text {CONT }}$ & $\begin{array}{c}\text { Limites } \\
\text { DNIT } \\
(2006 \mathrm{a})\end{array}$ & $\begin{array}{c}\text { MAE a } \\
\text { frio }\end{array}$ & PMF $_{\text {CONT }}$ & $\begin{array}{c}\text { Limites } \\
\text { DNIT } \\
(2010 \mathrm{a})\end{array}$ \\
\hline Estabilidade (kgf) & 161,62 & 1563 & $\geq 500$ & 351,87 & 335 & $\geq 250$ \\
\hline Fluência (mm) & 3,59 & 4,06 & - & 4,22 & 3,4 & 2 a 4,5 \\
\hline Vv (\%) & 8,73 & 3,57 & 3 a 5 & 9,26 & 15,6 & 5 a 30 \\
\hline RBV (\%) & 57,41 & 77,56 & 75 a 82 & 55,61 & 50,35 & - \\
\hline
\end{tabular}

Nos resultados da Tabela 8 fica claro que a mistura ensacada não se enquadra como uma mistura a quente de acordo com as exigências da especificação DNIT 31 (DNIT, 2006a). A estabilidade média apresentada pelo produto foi cerca de $10 \%$ do valor medido no CACONT e de $32 \%$ do mínimo exigido pela referida norma. Os valores de fluência foram similares entre essas misturas, entretanto, Vv e RBV da mistura ensacada ficaram consideravelmente fora dos limites da referida especificação. $\mathrm{Na}$ análise do produto como uma mistura a frio, contudo, a mistura ensacada apresentou valores de estabilidade fluência e Vv dentro dos limites estabelecidos na especificação DNIT 153 (DNIT, 2010a), com grandezas similares àquelas obtidas pelo PMFCONT. Ressalta-se que a mistura de controle do PMF foi estudada com granulometria dentro da faixa D (DNIT, 2010a), enquanto que a MAE ensaiada a frio não se enquadrou nessa faixa (Figura 3).

\subsection{Controle de qualidade da mistura ensacada em diferentes idades}

$\mathrm{Na}$ Tabela 9 são apresentados os valores médios de estabilidade, fluência, Vv e RT, dos testes realizados em triplicata, para energia de compactação de 75 golpes e idades de 30, 90, 180 e 360 dias de armazenamento da MAE, ensaiada como mistura a frio, além dos respectivos valores de CV.

Os resultados apresentados na Tabela 9 não indicam tendência de comportamento bem definida entre o tempo de estocagem da mistura produzida e os respectivos parâmetros avaliados. Nota-se que a estabilidade decresceu de 30 para 90 dias, voltando a subir na análise com 180 dias e novamente a decrescer com 360 dias, com elevados valores de CV. Com a fluência e RT o comportamento observado na estabilidade se repete, enquanto no volume de vazios, embora também não demonstre uma tendência clara, 
apresenta o menor coeficiente de variação entre os três parâmetros avaliados. Os elevados CVs observados no presente estudo corroboram os resultados obtidos por Santana et al. (2017) e Rezende et al. (2019), não sendo possível definir, com os dados obtidos, o tempo máximo de armazenamento em que o produto mantém as características mínimas exigidas pela especificação DNIT 153 (DNIT, 2010a). Tais resultados demonstram a necessidade de normatização das misturas ensacadas, de forma a estabelecer níveis de performance ao longo do tempo de armazenamento.

Tabela 9: Parâmetros médios de controle de qualidade nas diferentes idades da MAE

\begin{tabular}{ccccccccc}
\hline Idade & \multicolumn{2}{c}{ Estabilidade $(\mathrm{kgf})$} & \multicolumn{2}{c}{ Fluência $(\mathrm{mm})$} & \multicolumn{2}{c}{ Vv $(\%)$} & \multicolumn{2}{c}{ RT $\left(\mathrm{kgf} / \mathrm{cm}^{2}\right)$} \\
\hline (Dias) & Média & CV & Média & CV & Média & CV & Média & CV \\
\hline 30 & 284,57 & 5,95 & 4,91 & 11,95 & 17,67 & 1,08 & - & - \\
\hline 90 & 242,96 & 3,20 & 3,22 & 9,11 & 18,47 & 2,32 & 0,38 & 5,60 \\
\hline 180 & 344,29 & 7,52 & 4,06 & 0,00 & 17,76 & 1,72 & 0,90 & 13,40 \\
\hline 360 & 207,92 & 20,11 & 2,88 & 10,19 & 19,71 & 2,30 & 0,44 & 14,82 \\
\hline $\begin{array}{c}\text { Limites } \\
\text { DNIT }\end{array}$ & $\geq 250$ & & 2 a 4,5 & & 5 a 30 & & - & - \\
\hline
\end{tabular}

\section{CONCLUSÃO}

A mistura ensacada analisada não pode ser classificada como uma mistura a quente nem como mistura a frio para utilização em rodovias federais do ponto de vista granulométrico, com base nas especificações para CA e PMF existentes no DNIT.

Em relação ao comportamento mecânico, entretanto, apesar de não apresentar características que a enquadrem como concreto asfáltico, a mistura comercial apresentou valores condizentes a uma mistura a frio, atendendo aos parâmetros do DNIT para tal, quando ensaiada com menor tempo de armazenamento. Entretanto, quando testada como mistura a frio a partir de seis meses de idade, a mistura não atendeu aos requisitos mínimos de estabilidade em amostras compactadas com 75 golpes.

Dessa forma, caso a mistura se enquadre granulometricamente em uma das faixas do DNIT, com manutenção do comportamento mecânico observado com pouca idade ao longo do tempo, o produto estudado poderá ser enquadrado como pré-misturado a frio e utilizado em rodovias federais para qualquer nível de tráfego.

\section{REFERENCIAS BIBLIOGRÁFICAS}

ANUÁRIO CNT DO TRANSPORTE: Estatísticas Consolidadas, 2019, http://anuariodotransporte.cnt.org.br/2019/inicial. Acesso em 12/05/2020.

DEPARTAMENTO NACIONAL DE ESTRADAS DE RODAGEM - DNER, 1994(a). ME 53/1994, Mistura betuminosa porcentagem de betume, Rio de Janeiro, RJ, Brasil. 1994.

DEPARTAMENTO NACIONAL DE ESTRADAS DE RODAGEM - DNER, 1994(b). ME 107/1994, Mistura betuminosa a frio, com emulsão asfáltica - ensaio Marshall, Rio de Janeiro, RJ, Brasil. 1994.

DEPARTAMENTO NACIONAL DE ESTRADAS DE RODAGEM - DNER, 1994(c). ME 78/1994, Agregado graúdoAdesividade a ligante betuminoso, Rio de Janeiro, RJ, Brasil. 1994.

DEPARTAMENTO NACIONAL DE ESTRADAS DE RODAGEM - DNER, 1994(d). ME 4/1994, Material betuminoso determinação da viscosidade Saybolt-Furol a alta temperatura método da película delgada, Rio de Janeiro, RJ, Brasil. 1994.

DEPARTAMENTO NACIONAL DE ESTRADAS DE RODAGEM - DNER, 1994(e). ME 148/1994, Material betuminoso determinação dos pontos de fulgor e de combustão (vaso aberto Cleveland), Rio de Janeiro, RJ, Brasil. 1994. 
DEPARTAMENTO NACIONAL DE ESTRADAS DE RODAGEM - DNER, 1994(f). ME 117/1994, Mistura betuminosa determinação da densidade aparente, Rio de Janeiro, RJ, Brasil. 1994.

DEPARTAMENTO NACIONAL DE ESTRADAS DE RODAGEM - DNER, 1995. ME 43/1995, Mistura betuminosa a quente - Ensaio Marshall, RJ, Brasil. 1995.

DEPARTAMENTO NACIONAL DE ESTRADAS DE RODAGEM - DNER, 1998(a). ME 83/1998, Agregados - Análise granulométrica, Rio de Janeiro, RJ, Brasil. 1998

DEPARTAMENTO NACIONAL DE ESTRADAS DE RODAGEM - DNER, 1998(b). ME 54/1998, Equivalente de areia, Rio de Janeiro, RJ, Brasil. 1998.

DEPARTAMENTO NACIONAL DE ESTRADAS DE RODAGEM - DNER, 1998(c). ME 81/1998, Agregados - Determinação da absorção e da densidade de agregado graúdo, Rio de Janeiro, RJ, Brasil. 1998.

DEPARTAMENTO NACIONAL DE ESTRADAS DE RODAGEM - DNER, 1998(d). ME 84/1998, Agregado miúdo determinação da densidade real, Rio de Janeiro, RJ, Brasil. 1998.

DEPARTAMENTO NACIONAL DE INFRAESTRUTURA DE TRANSPORTES - DNIT, 2006(a). ES 31/2006, Pavimentos flexíveis - Concreto asfáltico, Rio de Janeiro, RJ, Brasil. 2006.

DEPARTAMENTO NACIONAL DE INFRAESTRUTURA DE TRANSPORTES - DNIT, 2006(b). EM 95/2006, Cimentos asfálticos de petróleo, Rio de Janeiro, RJ, Brasil. 2006.

DEPARTAMENTO NACIONAL DE INFRAESTRUTURA DE TRANSPORTES - DNIT, 2010(a). ES 153/2010, Pavimentação asfáltica - Pré- misturado a frio com emulsão cationica convencional, Rio de Janeiro, RJ, Brasil. 2010.

DEPARTAMENTO NACIONAL DE INFRAESTRUTURA DE TRANSPORTES - DNIT, 2010(b). ME 155/2010, Material asfáltico - Determinação da penetração, Rio de Janeiro, RJ, Brasil. 2010.

DEPARTAMENTO NACIONAL DE INFRAESTRUTURA DE TRANSPORTES - DNIT, 2010(c). ME 131/2010, Materiais asfálticos - Determinação do ponto de amolecimento - Método do Anel e Bola, Rio de Janeiro, RJ, Brasil. 2010.

DEPARTAMENTO NACIONAL DE INFRAESTRUTURA DE TRANSPORTES - DNIT, 2011. ME 158/2011, Mistura asfáltica - Determinação da porcentagem de betume em mistura asfáltica utilizando o extrator Soxhlet, Rio de Janeiro, RJ, Brasil. 2011.

DEPARTAMENTO NACIONAL DE INFRAESTRUTURA DE TRANSPORTES - DNIT, 2013. EM 165/2013, Emulsões asfálticas para pavimentação, Rio de Janeiro, RJ, Brasil. 2013.

DEPARTAMENTO NACIONAL DE INFRAESTRUTURA DE TRANSPORTES - DNIT, 2018. ME 136/2018, Determinação da resistência à tração por compressão diametral, Rio de Janeiro, RJ, Brasil. 2018.

DEPARTAMENTO NACIONAL DE PRODUÇÃO MINERAL,2018. Cadastro Nacional de Produtores de Brita / Coord. Geral Wagner Fernandes Pinheiro [et. al]; Equipe Técnica por Júlio Recuero e [et. al]; - Brasília: DNPM, 2018. 82 p.

DULAIMI, A., SHANBARA, H. K., AL RIFAIE, A., 2020. "The mechanical evaluation of cold asphalt emulsion mixtures using a new cementitious material comprising ground-granulated blast-furnace slag and a calcium carbide residue", Construction and Building Materials, vol. 250, pp. 1-13, Jul. 2020.

REZENDE, D. A. T., SILVA, J. P. S., CARVALHO, I. S., REZENDE, L. R., 2019. "Caracterização amostral de concreto asfáltico estocável por meio de ensaios laboratoriais", Anais do $33^{0}$ Congresso de Pesquisa e Ensino em Transportes da ANPET, vol. 1, pp. 1438-1449, Balneário Camboriú/SC, Nov. 2019.

SANTANA, A. C. S. T., LOURENÇO, V. M. Q., 2017. "Análise das propriedades físicas e mecânicas de mistura asfáltica usinada à quente ensacada para aplicação à frio", Anais do $34^{0}$ Congresso de Pesquisa e Ensino em Transportes da ANPET, vol. 1, pp. 1574-1585, Recife/PE, Nov. 2017.

SANTANA, R. R., SANTOS, R., CAVALCANTE, E. H., CARDOSO, G., 2018. "Estabilidade e adesividade de ligante asfalto modificado com blenda de borra oleosa de petróleo com borracha de pneu”, Revista Matéria, vol. 23, n. 1, Mar 2018.

SANTOS, K. P., LUCENA, A. E. F. L., LUCENA, L. C. F., SILVA, J. A A., COSTA, S. C. F. E., 2015. "Estudo da incorporação de argilas montmorilonitas em cimentos asfálticos de petróleo”, Revista Matéria, vol. 20, n. 2, pp. 501-513, Mar. 2015.

SENA NETO, P. G., AMORIM, E. F., INGUNZA, M. P. D., 2019. "Análises de dosagens de concreto asfáltico do tipo Pré Misturado a Frio (PMF) utilizando Resíduos da Construção e Demolição de obras (RCD)", Revista Matéria, vol. 24, n. 4, Nov. 2019.

XAVIER, M. F., FERREIRA, W. L. G., CASTELO BRANCO, V. T. F., 2020. “Avaliação do uso de cinzas de carvão mineral como melhorador de adesividade em misturas asfálticas”, Revista Matéria, vol. 25, n. 1, Abr. 2020. 